\title{
Design of Intelligent Car Based on Arduino Development Board
}

\author{
$\mathrm{Ru} \mathrm{Nie}^{1, \mathrm{a}}$ \\ ${ }^{1}$ College of Electronic information engineering, Guangzhou College of South China \\ University of Technology, Guangzhou \\ a52811055@qq.com
}

Keywords: Arduino; Intelligent car; Microchip.

\begin{abstract}
As the development of science and technology, the microchip technology has become closer relationship to our life which gives our life more convenient, not only the telephone but computers and digital cameras can't work without the microchip's development. "Arduino”, it's cheap and easy to develop but high performance. It also could be easily connected to kinds of sensors. It is really an open-source digital platform with easy to develop, including hardware and software (Arduino IDE). Now, in here, I chose the Arduino board and take full use of it to develop this intelligent car which one could get the date around to working automatic, and it show us the board potential and value.
\end{abstract}

\section{Introduction}

In the information age, along with the computer technology and chip technology progress, all kinds of technology products, including whether the computer, TV, air conditioning, mobile phone or camera, all can't do without chip technology development and progress. The chip from the early sixty or seventy's simple microcontroller, up to the present advanced processor, has become more and more powerful now. 51 single chip computer is already familiar to the people, learning electronic professional students basically of 51 single chip microcomputer is a required course for them. But with the growing demand for the performance of the chip, the requirements are also constantly improve. Structure of 51 MCU obsolete and backward performance has been increasingly which is unable to meet the people and students thirsting for knowledge, so a new hardware platform born at this time. It is simple development and convenience operation, low cost hardware circuit, very suitable for the development and design, it can be used to achieve variety of needs which were past complex steps function easily. This is the Arduino development platform, an open source platform, fit for students from deprived backgrounds to learn and use. Arduino is a Simple interface based on USB I/O interface board of the open source code (including 12 digital GPIO channel, 4 PWM output channel, 6 8 channel 10 bit ADC input). The hardware of the system mainly consists of an Arduino UNO control board, Arduino Xbee sensor expansion board, Bluetooth serial module and digital temperature sensor [1].

The smart car's design has the following functions: Firstly the hunting function, which is also go follow with the black line on the ground; Secondly the infrared obstacle avoidance function, to perceive the external environment so as to avoid obstacles by infrared sensor; Thirdly the ultrasonic distance measuring function, which used ultrasound to measure the distance sensor; Fourthly the BS18B20 temperature sensor, which used for measuring temperature; and finally the function of the car with infrared remote control.

\section{The hardware design of intelligent vehicle control system based on Arduino development board}

2.1 The overall design of intelligent car control system

2.1.1 The realization of hunting line function 
The car wants to finish line function, hoping that it can follow the black line to go so he first has to be able to "see" the black line, then here is to use the sensor, sensor, is a kind of detection device is simple, can feel the measured information, and the detected information, according to the law of transformation certain into electric signal or other forms of information required for the output, in order to satisfy the information transmission, processing, storage, display, record, control requirements. Here will be the selection of an infrared tour line sensor, and the infrared sensor will act as a smart car eyes to detect the black line, the principle of infrared line sensor module is to pipe detection oneself to emit infrared ray to the reflected light by infrared (dark reflection of weak, light reflecting strong). White or black for routing sensor can help your robot tracking, can detect the white in the black line, can also be white line detecting and black bottom, detect black line back to lower level.

Design of car is the use of pre drive, two left and right front wheel are respectively controlled by two motors, and the back is a universal wheel, can rotate freely. So when determining the car model, if the two front wheels car also forward at the same speed if the car to move forward. When car right wheel forward revolver don't understand so the car will turn left, turn right round revolver similarly trolley forward real then the car to the right, two wheels at the same time at the same speed as the car will reverse turn back.

The next step is composed of a sensor control trolley, a set of three line sensors here, three sensors will have three kinds of cases, respectively, each sensor is met the black line and two did not meet the black line of three cases. The middle one is to detect the black line, when a test in the middle to black returned to low level, this car go straight, when the black line to the left turn, at this time the car if the sensor is left go straight will detect black line, now is the time to return to the low level, right rotation, left wheel stop, car will turn to the left when to turn the corner on the left, after the sensor has been detected Apodoses, intermediate and can detect black line, then continue straight ahead. Similarly, when the sensor to turn right to the right of the black line will also detect black line, return to low level, at this time the car turn right round revolver, stop, and so the car would like to turn to the right, until the middle of the sensors detect black line so far.

\subsubsection{The realization of obstacle avoidance function}

As for the obstacle avoidance function trolley, first choice of sensor, here using infrared obstacle avoidance sensors, he is composed of an infrared transmitting tube and an infrared receiving tube, the principle of work is: transmitting infrared sensor, according to the reflected infrared light detection of forward obstacles, high level output without obstacles, output low level there are obstacles, so it's routing sensor principle of the sensor before and is about the same, so you can do the same, the first sensor is installed in the car's front end to feel the environment change, here like to use three sensors are respectively placed in front, front left, front right. So here a bit a bit more complex than in front of the line function, because the homing line only one, but here there may be only one sensor is blocked, there may be two sensors are blocked, there may be three sensors are blocked, so here we need to these kinds of situations are taken into account.

\subsubsection{The realization of ultrasonic distance measurement function}

For the ultrasonic ranging function, using HC-SR04, it is one of the most common ultrasonic sensor, the price is cheap, easy to use. The ultrasonic transmitting and receiving by HC-SR04 module, module comprises an ultrasonic transmitter, receiver and the control circuit [2]. Ultrasonic principle is very simple, want to achieve is a very convenient thing in the Arduino platform, but because you want to display on your computer, so don't forget to serial port baud rate setting.

\subsubsection{The realization of ultrasonic distance measurement function}

Temperature sensing function, here is a selection of digital temperature sensor DS18B20, DS18B20 is a kind of improved intelligent temperature sensor America DALLAS Semiconductor Company after DS1820's new [3], and DS18B20 digital temperature sensor is a single bus digital temperature sensor American production company DALLAS. DS18B20 has the advantages of simple temperature measuring system, high temperature measuring precision, convenient connection, occupy less interface line etc. [4], From the host CPU to the DSl820 only a line (and ground) DSl820 power supply can be provided by a data line without the need for external power supply for each 
DSl820 in the factory has been given a unique serial number so that any number of DSl820 can be stored in the same single line bus which allows the measurement range of temperature sensitive device DSl820 placement in many different places to 0.5 in the $1 \mathrm{~s}$ value to the +125 increments from -55 (typical value) within the temperature is converted into digital. DS18B20 temperature measurement principle of understanding simple is said to the chip to the perceived temperature change values in the data register inside, must want to obtain the register data inside, only in accordance with the provisions of a sequential DALLAS can correct outgoing data, this sequence is called the single bus, CPU can pass through a single bus protocol, which made DS18B20 the temperature value.

2.1.5 The Implementation of infrared control function

In order to realize the infrared control function, is selected the $38 \mathrm{~K}$ infrared receiving module, and also with a small remote control to facilitate control. Want to achieve the goal here is to control the car to walk, that is to control the vehicle forward, backward, turn left, turn right, how to achieve it. The front of the line or, infrared obstacle avoidance or, have provided a team of trolley control function, call in here, this is not a problem, limited to the essence of infrared emission, is the infrared, so in the control range is limited, just like the TV remote control home, must be aligned to the remote control, so the infrared receiving head is installed in the car behind, control in the back.

2.2 The structure diagram of intelligent car system

Figure 1 is the car by three sensor control operations, in addition to two sensors is the result of monitoring window to be output to the computer by software to monitor, as shown in figure 2.

\section{The software design of intelligent vehicle control system based on Arduino development board}

\subsection{Ultrasonic sensing program design}

Ultrasonic ranging sensor is then detected by first transmitting ultrasonic consumed returned ultrasonic time to compute the distance, below 3 is a flow chart.

3.2 Infrared remote control program design

Because the infrared module, equipped with a remote control, so read the values of each of the remote control button out, then each button value recorded, so will each value as an operation command, when the button is pressed in the remote, emit the corresponding value, then when the infrared receiving the first receive signal processor will read the value, and make the corresponding response according to the program, figure 4 is a diagram of the infrared control.

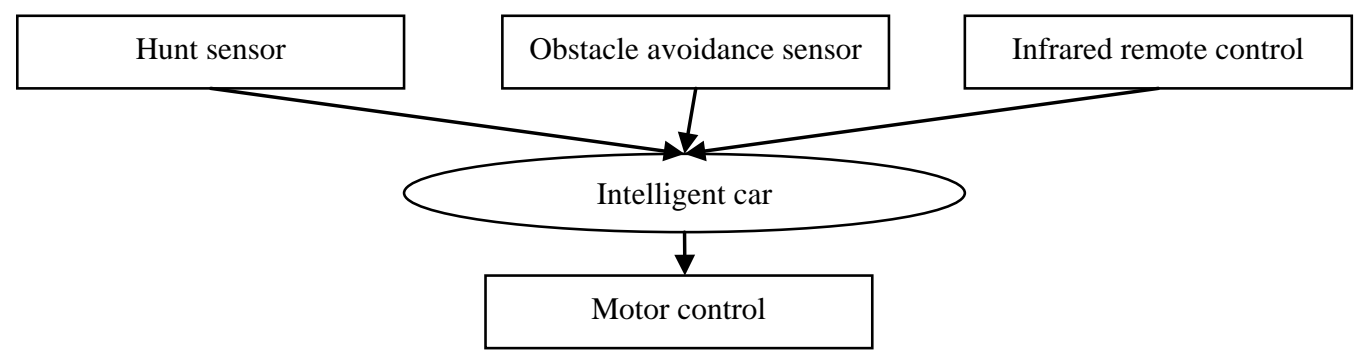

Fig.1 Control car block diagram 1

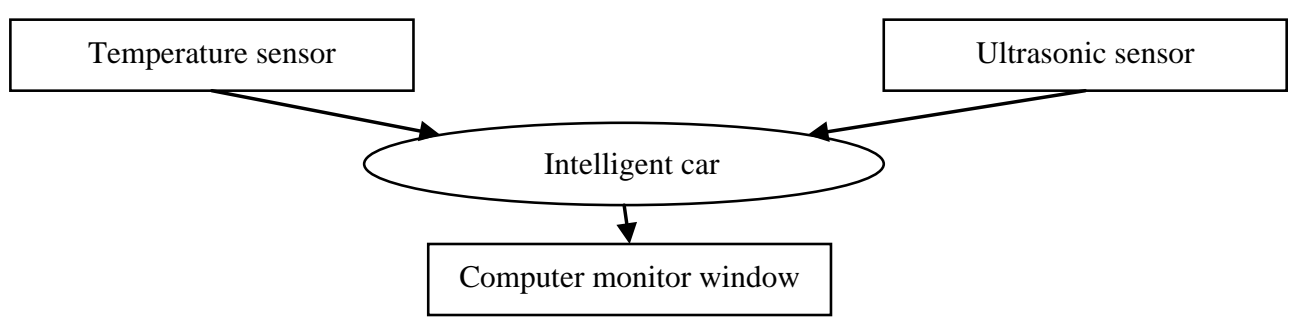

Fig. 2 Control car block diagram 2

The definition of various parameters and initialization 


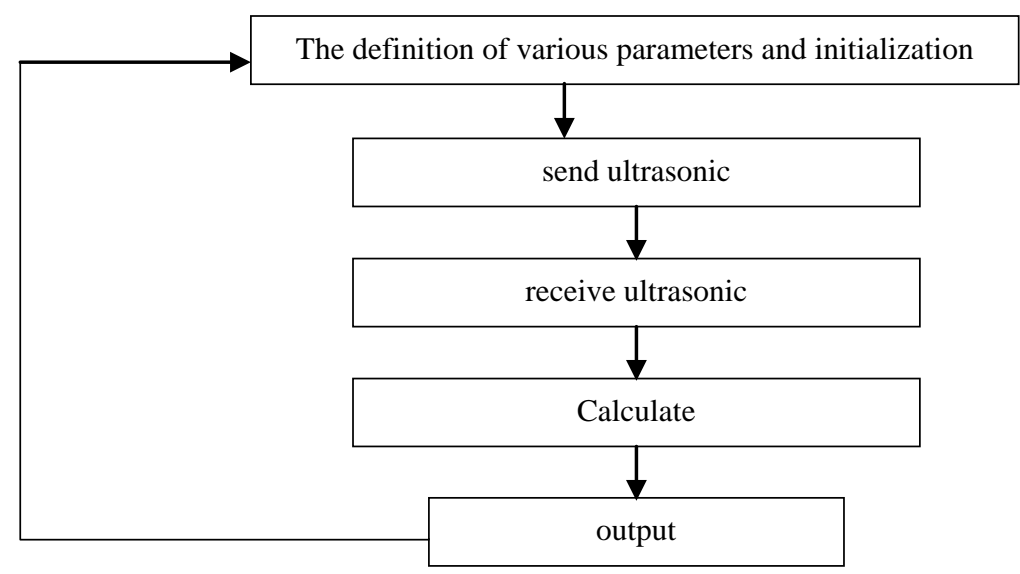

Fig. 3 Ultrasonic ranging chart

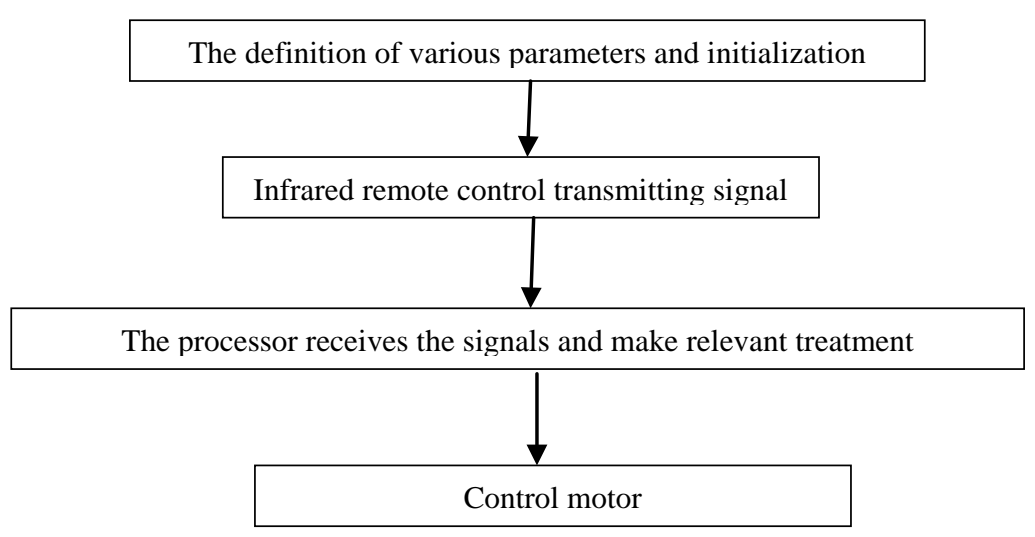

Fig. 4 Infrared remote control chart

\section{Conclusion}

On the designing of intelligent car system Arduino development board based on the hardware and software, realizes the automatic car tracking, obstacle avoidance, ultrasonic distance measurement functions. In the design of the software uses the modular design thought, the experimental results show that: the use of sensors to automatically collected environmental data which according to the program to run automatically. The operation of the smart car design is simple, stable and reliable, the system has broad potential and application value.

\section{References}

[1]Zheng Hao, ZHONG Zhifeng,GUO Hao. Design of Bluetooth communication system based on Arduino/Android [J]. Internet of Things Technologies, 2012， （5）:50-51.

[2]SU Lin. Design of ultrasonic range finder based on HC-SR04[J]. Science \& Technology Information, 2012, (9) : 124-125.

[3] ZHAO Hailan.Intelligent temperature sensor DS18B20[J]. Electronics World, 2003, （7）: 46-47.

[4] GAO Yunhong. Application of digital temperature sensor in multi-point temperature measurement system[J]. Journal of Shenyang Institute of Aeronautical Engineering, 2006,23 (2) : 61-63. 